\title{
Cell to cell hijacking: Role of membrane nanotubes
}

Cell to Cell communications is the pivot for life processes. Any event that disrupts this leads to the loss of physiological function, eventually leading to cell death. Evolutionarily, cells developed an elaborate mechanism to undertake this paramount responsibility through cell surface glycocalyx, receptors, integrins, and other recognition molecules. Cells also exchange through local acting soluble mediators as well as through vesicles and exosomes. Recent development in this field led to the identification of a spectacular network of membrane process that seems to be the supremo of all that was known about cellular communications. These are called membrane nanotubes or tunneling nanotubes (TNT). ${ }^{1}$ Cellular communication can be subdivided into contact and contactless. The former provides more rapid and molecule transfer as compared to the latter. TNTs are a novel type of contact-based communication. ${ }^{2}$ TNTs are straight, thin membrane extensions connecting cells over long distances first reported in PC12 cells in 2004. TNT is believed to form from actin-based membrane protrusion. There are three different models of TNT formation. (a) Protrusions from one cell grow and extend until it reaches the other cell, followed by a membrane fusion. (b) Membrane protrusions grow from both cells until they meet and establish a connection. (c) TNT formation by cell dislodgement when cells migrate further apart from each other, and during this movement, TNT is formed. ${ }^{3} \mathrm{It}$ is possible that all these three models may be operational depending on cell types and context.

TNT are dynamic connections between cells, representing a novel route for cell-to-cell communication (Figure 1). ${ }^{4}$ TNT was reported in various cell types, like epithelial cells, neuronal cells, mesenchyma cells, and immune cells engaged in intercellular exchanges of molecules, subcellular organelles, and pathogen and viruses transport routes. ${ }^{5}$ TNT can extend up to $200 \mu \mathrm{m}$ in length and about $50-1500 \mathrm{~nm}$ in diameter in macrophages. TNT can be established between similar cell types(homo-TNT) or between one cell type and another (hetro TNT) and thus may be involved in the initiation and growth of cancer as well as dissemination of cancer cells. ${ }^{6}$ TNTs are also assumed to play a role in treatment resistance, e.g., in chemotherapy treatment of cancer. Recently, TNT has been used to hijack mitochondria from healthy cells by the cancer cells as a source of energy. ${ }^{7} \mathrm{TNT}$ was also reported

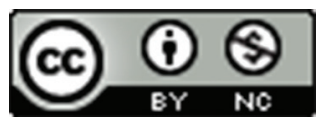

This work is licensed under a Creative Commons Attribution-NonCommercial 4.0 International License.

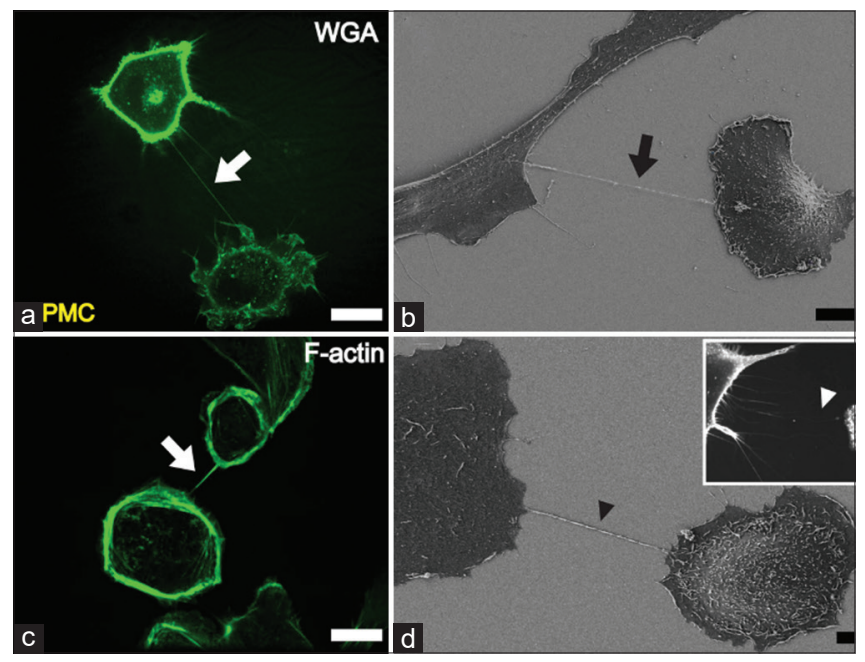

Figure 1: Membrane Nanotubes as seen (arrows) through confocal microscopy (a and c) and Scanning Electron Microscopy (b and d). Images were taken from Wikipedia (https://en.wikipedia.org/wiki/ Tunneling_nanotube)

to transport miRNA and other RNA to the surrounding stroma creating an environment suitable for cancer growth. ${ }^{8}$ More research in this discipline is needed to understand the full function of these wonderful nanostructures.

Original Publication and Credit to: Ranzinger J, Rustom A, Abel M, Leyh J, Kihm L, Witkowski M, et al. (2011) Nanotube Action between Human Mesothelial Cells 
Reveals Novel Aspects of Inflammatory Responses. PLoS ONE 6(12): e29537. https://doi.org/10.1371/journal. pone.0029537 https://commons.wikimedia.org/w/index. php?curid $=17826292$

\section{Karthikeyan Pethusamy', Ruby Dhar ${ }^{2}$, Arun Kumar ${ }^{3}$, Subhradip Karmakar ${ }^{4}$}

${ }^{1}$ Research Scholar, ${ }^{2}$ Scientist, ${ }^{4}$ Additional Professor, Department of Biochemistry, All India Institute of Medical Sciences, New Delhi, ${ }^{3}$ Professor, Department of Biochemistry, Jagannath Gupta Institute of Medical Sciences and Hospital, Budge Budge, Kolkata, India

Address for Correspondence:

Dr. Arun Kumar, Professor, Department of Biochemistry, Jagannath Gupta Institute of Medical Sciences and Hospital, Budge Budge, Kolkata, India. Mobile: +91-7584089886.

E-mail: arun732003@gmail.com

Dr. Subhradip Karmakar, Additional Professor, Department of Biochemistry, All India Institute of Medical Sciences, New Delhi, India. Mobile: +91-9999612564.

E-mail: subhradipaiims@gmail.com

\section{REFERENCES}

1. Sherer NM and Mothes W. Cytonemes and tunneling nanotubules in cell-cell communication and viral pathogenesis. Trends Cell Biol. 2008;18(9):414-420. https://doi.org/10.1016/j.tcb.2008.07.003

2. Gurke S, Barroso JF and Gerdes HH. The art of cellular communication: Tunneling nanotubes bridge the divide. Histochem Cell Biol. 2008;129(5):539-550.

https://doi.org/10.1007/s00418-008-0412-0

3. Gerdes $\mathrm{HH}$ and Carvalho RN. Intercellular transfer mediated by tunneling nanotubes. Curr Opin Cell Biol. 2008;20(4):470-475. https://doi.org/10.1016/j.ceb.2008.03.005

4. Rustom A, Saffrich R, Markovic I, Walther P and Gerdes HH. Nanotubular highways for intercellular organelle transport. Science. 2004;303(5660):1007-1010.

https://doi.org/10.1126/science.1093133

5. Sowinski S, Jolly $C$, Berninghausen $O$, Purbhoo MA, Chauveau A, Köhler K, et al. Membrane nanotubes physically connect $T$ cells over long distances presenting a novel route for HIV-1 transmission. Nat Cell Biol. 2008;10(2):211-219. https://doi.org/10.1038/ncb1682

6. Matejka N and Reindl J. Perspectives of cellular communication through tunneling nanotubes in cancer cells and the connection to radiation effects. Radiat Oncol. 2019;14(1):218

https://doi.org/10.1186/s13014-019-1416-8

7. Connor Y, Tekleab S, Nandakumar S, Walls C, Tekleab Y, Husain $A$, et al. Physical nanoscale conduit-mediated communication between tumour cells and the endothelium modulates endothelial phenotype. Nat Commun. 2015;6:8671.

https://doi.org/10.1038/ncomms9671

8. Saha T, Dash C, Jayabalan R, Khiste S, Kulkarni A, Kurmi K, et al. Intercellular nanotubes mediate mitochondrial trafficking between cancer and immune cells. Nat Nanotechnol. 2021. https://doi.org/10.1038/s41565-021-01000-4

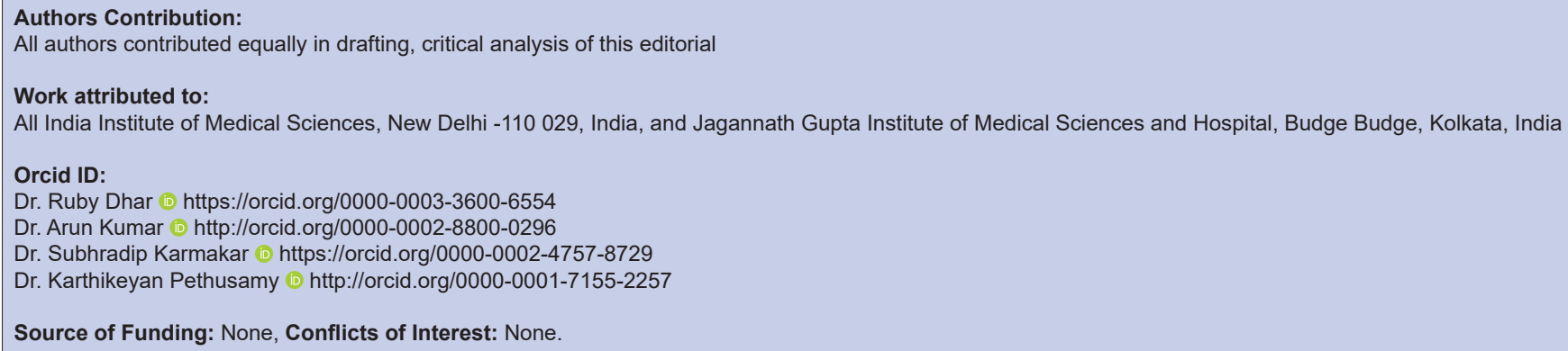

